\title{
The Analysis of Dimensionless Magnitudes in Economic Science
}

\author{
Odysseas Kopsidas \\ University of Piraeus, Piraeus, Greece
}

\begin{abstract}
An economy consists of the economic system of a country or other unit of human society. It includes labor, capital, natural resources, production, trade, distribution and consumption of goods and services in the area where human society is active. These factors give context, content, and determine the conditions and parameters with which the economy operates. When searching with data mining techniques to identify or find out dimensionless groups (DGs) in technical literature, it is possible to meet errors/faults/omissions concerning both, the form and the content of such groups. In the present study, a methodological framework has been developed in terms of a logical flow chart, including 11 activity stages and seven decision nodes, to acquire/process/store/retrieve knowledge for reconstruction and identification of these groups. Case Based Reasoning (CBR), especially modified to meet the needs of this work, has been used for tracing causality paths by similarity and making correction suggestions. Two case examples are presented to prove the functionality of the proposed methodology. Non-dimensional groups are used in engineering but can also be used in economic science. Through this analysis, we can calculate the scale of industrial processes from laboratory to pilot and then factory scale. Still through the study of non-dimensional groups, it is easy to calculate economies of scale embedded in the production process. Synergy savings and target economies cause economies of scale in a production process and reduce the cost of production per unit of output when production is increased. Non-dimensional groups can be a quantitative and measurable indicator for calculating and predicting economies of scale in an industrial unit. The same can happen in an economic unit providing services, that is, intangible products.
\end{abstract}

Keywords: dimensional analysis, knowledge acquisition, dimensionless groups, case based reasoning, data mining, Blake number, modified Reynolds number, adsorption, sedimentation, flocculation, filtration, scale up

\section{Introduction}

Some sizes are defined as the quotient of two identical sizes and so they are dimensionless or in other words they have a dimension that can be expressed by the number "1". The associated SI unit for all dimensionless sizes, dimensions one, is number one, since the quotient of two SI units must be equal to one. The values of these sizes are simply expressed as numbers and the unit "1" is not next to them. Examples of such sizes are diffraction index, relative permeability, coefficient of friction, and so on.

In very few cases, however, separate names have been given to unit " 1 " to facilitate the separation of the

Odysseas Kopsidas, Ph.D., Department of Industrial Management and Technology, University of Piraeus, Piraeus, Greece.

Correspondence concerning this paper should be addressed to Odysseas Kopsidas, Department of Industrial Management and Technology, University of Piraeus, Karaloli \& Dimitriou 80, Piraeus 18534, Greece. 
relevant sizes. This applies to the radian and steering. The radius and steering are recognized as special names for the associated SI units used to express the flat angle and solid angle values, respectively. In all these cases of counts the SI unit is considered to be the number one. The number one in these cases cannot be considered as a unit of production and therefore for these cases the number one can be considered as one additional basic SI unit.

Dimensional analysis is applied in those cases of flow of fluids that do not require a precise theoretical solution, or their mathematical solution is not possible.

Thus, in these cases the experimental study is followed where the dimensional analysis is applied in which the plurality of different sizes make up a limited number of non-dimensional groups for ease of reference.

Dimensional analysis was initially developed early in the last century (Rayleigh, 1900; 1915; Buckingham, 1914; Langahaar, 1951; Huntley, 1952; Palacios, 1956) by scientists and engineers for expressing the behavior of a physical system in term of the minimum number of independent variables and in a form that is unaffected by changes in the magnitude of the units of measurement. The first attempt to a similar approach in Economics was made by Allais (1943), who presented a systematic treatment of the theory of dimensions and its foundations. Later, some authors contributed to the diffusion of this scientific topic (e.g., see Jong, 1967) or used relevant techniques either for simply stressing the distinction between stock and flow variables (Boulding, 1966; Auckley, 1961) or for techno-economic analysis (Nussbaumer \& Neuenschwander, 2000; Corre, Guillaum, \& Mohand, 2002) or for model checking (Batzias \& Sidiras, 2004). The physical and/or economic magnitudes are usually arranged in dimensionless groups (DGs), the number of which is $m=n-r$, where $n$ is the number of dimensional variables/parameters/constants (VPCs) and $r$ is the number of primary quantities used, like mass M, length L, time T, and temperature G. This rule is a general relation based on Linear Algebra and Group Theory and is mostly quoted as Buckingham's pi theorem.

In differential analysis, the fluid control volume, as above but in infinite dimensions where the principles of conservation of mass, momentum, and energy are also applied.

Thus, similarly with their application, the differential equations of their continuity arise, the solution of which, by using marginal initial conditions, provides precise values, or even distribution of fluid properties, such as e.g. speed, pressure, shear stress, etc. From these, it is even possible to calculate the maximum values of these sizes.

DGs may have physical meaning and in such a case we can search for similar groups through Case Based Reasoning (CBR) in order to properly describe quantitatively and solve similar problems, possibly occurring due to errors either in dimensions or in form or in content. CBR has been formalized for purposes of computer-aided processing as a four-step algorithmic procedure, which might be named 4R from the initial letter of the four verbs used as computer program commands in the corresponding steps: (I) Retrieve the cases considered to be relevant to solving a given target problem; (II) Re-use the solution (if it was successful) from the previous case to the target problem by making the necessary modifications to adapt the solution as needed to fit the new situation; (III) Revise by testing the solution of the new problem directly in the real world or indirectly under suitable conditions or reliable simulation; (IV) Retain by storing the solution as a new case in the computer memory, provided that the adaptation was a successful one.

Computer-aided solving by CBR the problems quoted above is a difficult task, especially when this approach uses unpublished evidence as its main Knowledge Base (KB); since the available information from the past may be not relevant/reliable to the required level for backing a successful 
"implementation-by-analogy", there is no guarantee that the necessary generalization (that ensures applicability) is correct. Nevertheless, inductive reasoning in case of scarce data/information for statistical relevance/reliability is usually based on incomplete evidence in practice. Moreover, there is always the possibility of developing CBR within a statistical framework and formalizing case-based inference as a specific type of probabilistic/possibilistic inference; therefore, it becomes feasible to make case-based estimates with a preset level of confidence.

\section{Methodology}

The methodological framework we have developed, in terms of a logical flow chart with 11 activity stages and seven decision nodes (interconnected as shown in Figure 1), to acquire/process/store/retrieve knowledge for reconstruction and identification of dimensionless groups by means of CBR, is presented below:

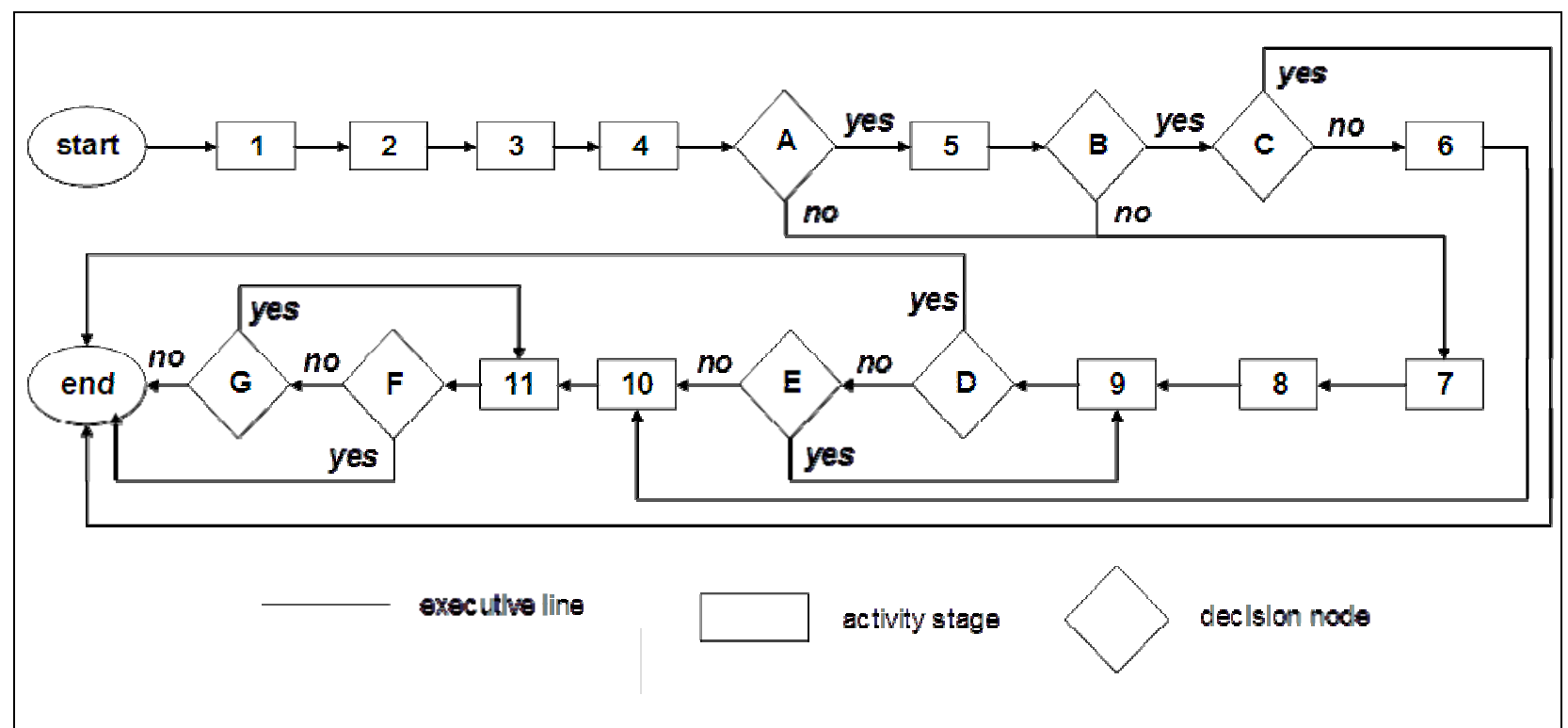

Figure 1. The logical flow chart developed for acquiring/processing/storing/retrieving knowledge for identification and correction of either dimensionally inconsistent VPCs or/and DGs in error.

(1) Analysis of the DG in error or the dimensionally inconsistent VPC.

(2) Checking of the definitions of the VPCs registered under widely known names in relevant KBs and, subsequently, in the corresponding original sources of technical literature.

(3) Translation of these verbal or symbolic definitions into dimensional definitions in accordance with the dimensional system adopted from the start as most appropriate.

(4) Replacement of the VPCs suspected to be in error with the new/checked ones and testing of the DG under consideration for dimensional homogeneity.

(5) Checking for operability within a proper/successful paradigm extracted from a KB by means of data mining and knowledge acquisition techniques to be used in accordance with CBR.

(6) Combinations of DGs to find out those which are more close to the DG under consideration.

(7) Checking of these combinations for operability ab initio (i.e., without having necessarily a past successful paradigm).

(8) Discrimination of relevant DGs and ranking of them in descending order of similarity degree as 
regards the DG to be corrected.

(9) DA by replacing a priori the DG under correction with the most similar (but unexamined so far) DG within the set of DGs resulted as the solution of the dimensional matrix corresponding to the system of equations obtained according to Rayleigh's Method of Indices.

(10) Multicriteria ranking of combinations performed in stage 6, according to the order of decreasing degree of closeness to the DG under consideration.

(11) Checking of the first combination (among the ones not examined so far) for operability.

(12) Has dimensional homogeneity been restored?

(13) Is operability satisfactory?

(14) Is the new DG identified as the correction of the old one?

(15) Is this replacement successful?

(16) Is there another ranked DG with lower similarity degree?

(17) Is its operability satisfactory?

(18) Is there another ranked combination not examined?

\section{Implementation}

We have implemented successfully the methodology described above in a plethora of cases. Subsequently, we present two of them. The first refers to modified Reynolds or Blake Number, defined in Perry's Chemical Engineering Handbook (Perry \& Green, 1997) as $B=V \rho /[\mu(1-\varepsilon) S]$, where $v$ : characteristic or average fluid velocity $(\mathrm{m} / \mathrm{s}) ; \rho$ : fluid density $\left(\mathrm{kg} / \mathrm{m}^{3}\right) ; \mu$ : fluid viscosity $(\mathrm{Pa} \mathrm{s}) ; \varepsilon$ : void fraction $\left(\mathrm{m}^{3}\right)$; $S$ : particle area/volume $(1 / \mathrm{m})$; all magnitudes measured in SI units. By replacing the units with the corresponding dimensions, we obtain $\mathrm{B} \in[\mathrm{LT}-1]$ [ML-3][ML-1T-1]-1[L3]-1[L2L-3]-1 or B $\in[\mathrm{L}-3] \neq[1]$. Evidently, this is in error, since the result should be $\mathrm{B} \in[1]$, i.e., dimensionless. Searching in external KBs by means of data mining and knowledge acquisition techniques incorporated within an Intelligent Agent (IA), according to Batzias and Marcoulaki (2002), we find the most recent reference source (Batzias, Kakos, \& Nikolaou, 2005) with all common DGs, where $\mathrm{B}=\mathrm{V} \rho /[\mu(1-\varepsilon) \mathrm{d}]$, with $\varepsilon \in[1]$, while $\mathrm{d}$ is the characteristic particle diameter $\mathrm{d} \in[\mathrm{L}]$ as recognized by the computer aided DA procedure adopted herein and thoroughly described in Walker and Weatherley (2001). Working out as above, we obtain B $\in$ $[\mathrm{LT}-1][\mathrm{ML}-3][\mathrm{ML}-1 \mathrm{~T}-1]-1[\mathrm{~L}]-1[\mathrm{~L} 2 \mathrm{~L}-3]-1$ or $\mathrm{B} \in[\mathrm{L}-1] \neq[1]$. By continuing the automatic searching, as shown in the flow chart of Figure 1, the computerized procedure identified finally the correct dimensions to be $\varepsilon \in[1]$ and $\mathrm{S} \equiv \mathrm{d} \in[\mathrm{L}-1]$, giving $\mathrm{B} \in[1]$.

The second case example used for implementation is the Freundlich isotherm, which we have used recently (Costa \& Marquez, 1998) to study by experimental simulation the dye adsorption in batch and fixed-bed systems:

$$
\mathrm{Q}=\mathrm{KC} 1 / \mathrm{n}
$$

where $\mathrm{q}=$ the amount of mass adsorbed per unit mass of the adsorbent [1]; $\mathrm{C}=$ the equilibrium concentration of the adsorbate, [ML-3]; $\mathrm{K}$ = parameter related to adsorption capacity; $\mathrm{n}$ = parameter related to adsorption intensity, [1]. Most authors do not specify the dimensions of K, while several of the rest of them assign either [L3M-1] or [1] (e.g., see Ng, Losso, Marshall, \& Rao, 2002 or Bird, Stewart, \& Lightfoot, 1960, respectively). Evidently, these dimensional assignments are in error, as the dimensional homogeneity of this isotherm requires 
$\mathrm{K} \in[\mathrm{M}-1 / \mathrm{n} \mathrm{L3/n}]$, although this assignment is meaningless, when its physical content is considered thoroughly. By referring mass $\mathrm{M}$ to dye in solution, dye adsorbent, adsorbent itself, we can split this primary dimension to $\mathrm{Ms}, \mathrm{Md}$, Mt, respectively. Consequently, we can write the isotherm model in dimensional form as follows: $\mathrm{q}=$ $\mathrm{Ks} \mathrm{Kd} \mathrm{Kt} \mathrm{C1/n,} \mathrm{where} \mathrm{q} \in[\mathrm{Md} \mathrm{Mt}-1], \mathrm{C} \in[\mathrm{Ms} \mathrm{L}-3], \mathrm{Ks} \in[\mathrm{Ms}-1 \mathrm{~L} 3]$ 1/n , Kd $\in[\mathrm{Md}], \mathrm{Kt} \in$ [Mt-1], which is meaningful as representing the real situation (facilitating also the scale up/down of the corresponding simulation procedures).

\section{Discussion}

Even in physical sciences and engineering/technology, this identification attempt becomes cumbersome and the computer program may fail to suggest a realistic solution without substantial human intervention. The probability to fail increases when the DG under investigation is incorporated into relations that change the original VPCs with others for sake of better fitting to system's specifications. For example, when the system is "fluid flow through porous media", B can be combined with the "resistance coefficient" DG expressed by the ratio $\psi=\mathrm{A}^{2} \varepsilon^{3} \mathrm{P} /\left[\mathrm{Q}^{2} \rho(1-\varepsilon) l \mathrm{~S}\right]$ to give a relation of the general form $\psi=\mathrm{K} / \mathrm{B}^{\mathrm{n}}$ in the procedure known as the Blake-Carman correlation, where $\mathrm{A}$ is the cross-sectional area of packing perpendicular to flow direction, $\mathrm{P}$ is the pressure difference, $\mathrm{Q}$ is the volumetric flow rate $(\mathrm{Q}=\mathrm{VA}), \quad l$ is the length of packing in the flow direction, $\mathrm{K}$ and $\mathrm{n}$ are parameters. In the low B-range (approximately, $\mathrm{B}<1$ ), $\mathrm{n}=1$, and by re-arranging the VPCs, we obtain the Kozeny-Carman equation $Q=A \varepsilon^{3} \mathrm{P} /\left[\mathrm{K} \mu(1-\varepsilon)^{2} \mathrm{~S}^{2} \mathrm{l}\right]$, which is dimensionally homogenous, since $\mathrm{Q} \in[\mathrm{L} 3 \mathrm{~T}-1]$ and $A \varepsilon^{3} \mathrm{P} /\left[\mathrm{K} \mu(1-\varepsilon)^{2} \mathrm{~S}^{2} \mathrm{~L}\right] \in[\mathrm{L} 2][1][\mathrm{ML}-1 \mathrm{~T}-2][1][\mathrm{ML}-1 \mathrm{~T}-1][\mathrm{L}-1]-2[\mathrm{~L}]-1$ $=[\mathrm{L} 3 \mathrm{~T}-1]$.

In such a case, we cannot investigate any dimensional inhomogeneity in B since this DG does not appear per se in the final model while the investigation of any error propagation should be based on CBR where the similar cases to be retrieved should belong to a broader family of Kozeny-Carman equations. If, according to Batzias, Kakos, and Nikolaou (2005), S is replaced by d in B, then the Kozeny-Carman equation becomes $\mathrm{Q}=\mathrm{A} \varepsilon^{3} \mathrm{P} /\left[\mathrm{K} \mu(1-\varepsilon)^{2} \mathrm{Sdl}\right]$. Consequently, the dimensional vector of the right-hand side of this equation becomes [LT-1] which is inconsistent with the left hand-side dimensional vector [L3T-1] denoting the correct dimensions of volumetric flow rate Q. The IA cannot identify the source of error unless provided with further information corresponding to ancestor relations. On the other hand, the inference engine searching within the KB may identify Q with the "superficial velocity" $u_{0}$ (i.e., the average linear velocity the fluid would have in the column if no packing were present) quoted as the dependent variable of Blake-Kozeny equation in the classic work (Batzias \& Marcoulaki, 2002).

As a matter of fact, searching for the possible routes of error propagation and the corresponding implications is a "direct problem" while searching for the possible source of error through an identification procedure is the "inverse problem". In the case examined herein, searching for implications of error in Blake Number B is the direct problem while searching for the possible source of error in Kozeny-Carman equation is the inverse problem. It is also worthwhile noting that the same family of equations is frequently met under all pairwise combinations (i.e., Kozeny-Carman, Blake-Carman, Blake-Kozeny) leading to additional difficulties faced by the IA searching in external KBs using the keywords interface suggested in Batzias and Marcoulaki (2002).

\section{Conclusion}


In conclusion, we have indicated the functionality of the methodological framework presented herein by analyzing two simple cases, one for DG and another for VPC. If the DGs or VPCs in error have been incorporated/combined into/with other expressions, traceability decreases and human intervention, at least for changing the searching pattern through CBR, increases the effectiveness of computer aided performance while facilitates the KB's enrichment/restructuring.

In the past, economic activity was considered to be limited by natural resources, labor, and capital. This view, however, ignores the value of technology (which often means automation, speeding up processes, and lower operating costs) and innovation (which means new products, services, processes, and markets, but also expansion of markets, diversification of markets , niche markets, and increased revenue from various functions), especially those that produce intellectual property. A given economy is the result of a set of processes including culture, values, education, technological development, history, social organization, political structure, legal systems, geography, natural resources, and ecology of the environment, as main factors. These factors give context, content, and a set of conditions and parameters on the basis of which a given economy operates. An economy consists of the economic system of a country or other unit of human society. It includes labor, capital, natural resources, production, trade, distribution and consumption of goods and services in the area where human society is active. These factors give context, content, and determine the conditions and parameters with which the economy operates.

Economies of scale are a term of finance, referring to the reduction of costs that an enterprise achieves by increasing the quantity of the product produced and characterizing the production of any product. Although at first sight it seems strange, economies of scale are observed in the production of most products. This also leads to a clear trend of business growth and concentration of production on a few and very large players.

Non-dimensional groups are used in engineering but can also be used in economic science. Through this analysis, we can calculate the scale of industrial processes from laboratory to pilot and then factory scale. Still through the study of non-dimensional groups, it is easy to calculate economies of scale embedded in the production process. Synergy savings and target economies cause economies of scale in a production process and reduce the cost of production per unit of output when production is increased. Non-dimensional groups can be a quantitative and measurable indicator for calculating and predicting economies of scale in an industrial unit. The same can happen in an economic unit providing services, that is, intangible products.

\section{References}

Allais, M. (1943). Traite d' economie pure. Vol. I, Les Donnees generals de l' economie pure, Paris and vol. IV, Annexes. Auckley, G. (1961). Macroeconomic theory. New York.

Batzias, F. A., \& Sidiras, D. K. (2004). Dye adsorption by calcium chloride treated beech sawdust in batch and fixed-bed systems. Journal of Hazardous Materials, 114, 167-174.

Batzias, F. A., \& Marcoulaki, E. C. (2002). Restructuring the keywords interface to enhance CAPE knowledge acquisition via an intelligent agent. Computer Aided Chemical Engineering, 10, 829-834.

Bird, R. B., Stewart, W. E., \& Lightfoot, E. N. (1960). Transport phenomena (pp. 196-200). New York: Wiley.

Boulding, K. E. (1966). Economic analysis (4th ed., Vol. II). New York.

Costa, C., \& Marquez, M. C. (1998). Kinetics of the PACT process. Water Research, 32, 107-114.

Huntley, H. E. (1952). Dimensional analysis. London, Mac Donald.

Jong, F. J. (1967). Dimensional analysis for economists. Amsterdam: North Holland Publ. Co.

Langahaar, H. E. (1951). Dimensional analysis. London. 
Ng, C., Losso, J. N., Marshall,W. E., \& Rao, R. M. (2002). Physical and chemical properties of selected agricultural byproduct-based activated carbons and their ability to adsorb geosmin. Bioresource Technology, 84(2), 177-185.

Nussbaumer, T., \& Neuenschwander, P. (2000). A new method for an economic assessment of heat and power plants using dimensionless numbers. Biomass Bioenerg, 18, 181-188.

Palacios, J. (1956). Dimensional analysis. London: Mac Millan.

Perry, R., \& Green, D. (1997). Perry’s chemical engineers' handbook (7th ed., pp. 648-650). McGraw-Hill.

Walker, G. M., \& Weatherley, L. R. (2001). Adsorption of dyes from aqueous solution-The effect of adsorbent pore size distribution and dye aggregation. Chemical Engineering Journal, 83, 201-206. 